\title{
Efficacy of Trifluralin Compared to Ethalfluralin Applied Alone and Co-Applied with Halosulfuron for Weed Management in White Bean
}

\author{
Nader Soltani*, Christy Shropshire, Peter H. Sikkema \\ University of Guelph Ridgetown Campus, Ridgetown, Canada \\ Email: *soltanin@uoguelph.ca
}

How to cite this paper: Soltani, N., Shropshire, C. and Sikkema, P.H. (2020) Efficacy of Trifluralin Compared to Ethalfluralin Applied Alone and Co-Applied with Halosulfuron for Weed Management in White Bean. Agricultural Sciences, 11, 837-848. https://doi.org/10.4236/as.2020.119053

Received: August 25, 2020

Accepted: September 24, 2020

Published: September 27, 2020

Copyright $\odot 2020$ by author(s) and Scientific Research Publishing Inc. This work is licensed under the Creative Commons Attribution International License (CC BY 4.0).

http://creativecommons.org/licenses/by/4.0/

(c) (i) Open Access

\begin{abstract}
There are a limited number of herbicides registered for weed management in white bean production in Ontario, Canada. Five field experiments were completed in Ontario from 2016 to 2018 to compare the efficacy of trifluralin and ethalfluralin applied alone and in combination with halosulfuron, applied preplant incorporated (PPI), for weed control efficacy and white bean tolerance and seed yield. At 2 and $4 \mathrm{WAE}$, there was no white bean injury from the herbicide treatments evaluated. Trifluralin applied PPI provided up to $32 \%, 99 \%, 13 \%, 99 \%, 27 \%, 99 \%$ and $99 \%$ control of velvetleaf, redroot pigweed, common ragweed, common lambsquarters, wild mustard, barnyardgrass and green foxtail, respectively. Trifluralin and ethalfluralin provide similar control of velvetleaf, redroot pigweed, barnyardgrass and green foxtail control, however, ethalfluralin is slightly more efficacious on common ragweed, common lambsquarters and wild mustard. Halosulfuron (35 $\mathrm{g} \cdot \mathrm{ai} \cdot \mathrm{ha} \mathrm{a}^{-1}$ ), applied PPI, provided as much as $76 \%, 98 \%, 96 \%, 96 \%, 100 \%, 19 \%$ and $23 \%$ control of velvetleaf, redroot pigweed, common ragweed, common lambsquarters, wild mustard, barnyardgrass and green foxtail, respectively. Trifluralin (600 or $\left.1155 \mathrm{~g} \cdot \mathrm{ai} \cdot \mathrm{ha}^{-1}\right)+$ halosulfuron $\left(35 \mathrm{~g} \cdot \mathrm{ai} \cdot \mathrm{ha}^{-1}\right)$, applied PPI, provided up to $88 \%, 100 \%, 98 \%, 100 \%, 100 \%, 99 \%$ and $98 \%$ control of velvetleaf, redroot pigweed, common ragweed, common lambsquarters, wild mustard, barnyardgrass and green foxtail, respectively. Ethalfluralin (810 or $\left.1080 \mathrm{ai} \cdot \mathrm{ha}^{-1}\right)+$ halosulfuron ( $35 \mathrm{~g} \cdot \mathrm{ai} \cdot \mathrm{ha}^{-1}$ ) provided similar control. Weed interference decreased white bean seed yield $44 \%$ - 45\% with trifluralin, 30\% $41 \%$ with ethalfluralin and $34 \%$ with halosulfuron. However, decreased weed interference with trifluralin and ethalfluralin applied in combination with halosulfuron resulted white bean seed yield that was similar to the weed-free
\end{abstract}


control. Trifluralin or ethalfluralin co-applied with halosulfuron can be safely used in white bean production for the control of common annual grass and broadleaf weeds in Ontario.

\section{Keywords}

ALS Inhibitor Herbicides, Crop Injury, Dinitroanaline Herbicides, Navy Bean, Phaseolus vulgaris, Sulfonylurea Herbicides

\section{Introduction}

Canada is one of the major dry bean (Phaseolus vulgaris L.) producing countries in the world [1]. White (navy) bean is the largest market class of dry bean grown in Canada [2]. Most of the white bean produced in Canada is grown in Ontario. On an annual basis, white bean growers in Ontario harvest approximately 26,000 hectares and produce 58,000 tonnes of white bean with a farm gate value of $\$ 36,000,000$ [2]. Weeds management is a critical component of successful white bean production as weed interference can reduce seed yield as much as $81 \%$ [1] [3] [4] [5] [6]. There are a limited number of herbicides registered for weed management in white bean production in Ontario [7]. New herbicides/tank mixes are needed to effectively control common annual grass and broadleaf weeds in white bean production.

Halosulfuron [methyl 3-chloro-5-[(4,6-dimethoxypyrimidin-2-yl)carbamoylsulfamoyl]-1-methylpyrazole-4-carboxylate] is a Group 2 sulfonylurea herbicide that inhibits the acetolactate synthase (ALS) enzyme which is crucial for the production of isoleucine, valine and leucine, three branched-chain amino acids needed for protein synthesis within plants [8] [9]. Halosulfuron, provides residual control of annual broadleaf weeds including redroot pigweed (Amaranthus retroflexus L.), jimsonweed (Datura stramonium L.), ladysthumb (Persicaria maculosa Gray.), common lambsquarters (Chenopodium album L.), wild mustard (Sinapis arvensis L.), velvetleaf (Abutilon theophrasti Medic.) and common ragweed (Ambrosia artemesiifolia L.) [7] [8]. However, halosulfuron applied alone does not adequately control grass weed species and needs a grass herbicide partner for broad-spectrum weed control [7] [8].

Ethalfluralin [N-ethyl-N-(2-methylprop-2-enyl)-2,6-dinitro-4-(trifluoromethyl)aniline] is a Group 3 dinitroanaline herbicide that can control/suppress problematic grassy weeds such as barnyardgrass (Echinochloa crusgalli (L.) Beauv.), green foxtail (Setaria viridis L.), yellow foxtail (Setaria pumila L.), volunteer barley (Hordeum vulgare L.), volunteer wheat (Triticum aestivum L.) and wild oats (Avena sativa L.) [9] [10]. Ethalfluralin can also control/suppress broadleaf weeds such as kochia (Bassia scoparia subsp. densiflora), wild buckwheat (Polvgonum convolvulus L.), redroot pigweed and common lambsquarters [10] [11]. Ethalfluralin, a Group 3 herbicide, can be one component of a di- 
versified herbicide resistance management program to reduce the selection pressure for the evolution of herbicide-resistant weeds including wild oats, green foxtail and kochia [10]. Ethalfluralin is a microtubule polymerization inhibitor that reduces root elongation, causes swollen root tips, swelling of stems, and dark green or purpling of tissues in susceptible weeds [10]. Ethalfluralin does not control emerged weeds and should be applied preplant (PP), preplant incorporated (PPI) or preemergence (PRE) for the control of susceptible weeds. When applied PP, PPI or PRE, ethalfluralin controls susceptible weed species prior to emergence [10].

Trifluralin [2,6-dinitro-N,N-dipropyl-4-(trifluoromethyl)aniline] is another Group 3 dinitroanaline herbicide that controls most annual grasses and small-seeded annual broadleaf weeds such as redroot pigweed and common lambsquarters, including the triazine-tolerant biotypes [7] [8]. Trifluralin is registered as a soil-applied herbicide in many crops including soybean, dry bean, snap bean and lima bean [7] [12]. Halosulfuron has been recently registered at 25 to 50 g.ai-ai.ha ${ }^{-1}$ for use in white bean production [7]. Ethalfluralin is not currently registered for use in any market class of dry bean in Ontario. Ethalfluralin has been reported to be a more active herbicide than trifluralin [10]. Ethalfluralin and trifluralin co-applied with halosulfuron can provide effective broad-spectrum control of common annual grass and broadleaf weeds in white bean production in Ontario. However, limited information is available on comparing the efficacy of trifluralin and ethalfluralin applied alone and in combination with halosulfuron for weed management in white bean under Ontario environmental conditions.

The purpose of this study was to compare the efficacy of trifluralin and ethalfluralin applied alone and in combination with halosulfuron, applied PPI, on white bean tolerance and yield, and weed control efficacy.

\section{Materials and Methods}

\subsection{Experimental Methods}

Field experiments (total of 5) were carried out at the University of Guelph Ridgetown Campus, Ridgetown, ON, in 2016, 2017 and 2018, and at the Huron Research Station, University of Guelph, Exeter, ON, in 2017 and 2018. Each experiment was arranged in a randomized complete block design (RCBD) with 4 replications. Treatments evaluated are presented in Tables 1-8. The experimental plots were $3.0 \mathrm{~m}$ wide and 8 or $10 \mathrm{~m}$ long depending on lacation. White bean "T9905" was seeded approximately $4 \mathrm{~cm}$ deep at the rate of approximately 250,000 seeds $\mathrm{ha}^{-1}$ in rows that were spaced $75 \mathrm{~cm}$ apart in late May to early June.

Herbicides treatments were applied 1 - 2 days before seeding and incorporated within one day with two passes of a field cultivator with rolling basket harrows in opposite directions. Herbicides were applied with a $\mathrm{CO}_{2}$-pressurized backpack sprayer calibrated to deliver $200 \mathrm{~L} \cdot \mathrm{ha}^{-1}$ at $240 \mathrm{kPa}$. The spray boom was $1.5 \mathrm{~m}$ 
Table 1. Percent seed moisture content at maturity and yield for white bean treated with trifluralin or ethalfluralin and halosulfuron applied PPI at Exeter (2017-2018) and Ridgetown, ON, Canada (2016-2018). , $^{\text {b }}$

\begin{tabular}{cccc}
\hline Treatment & $\begin{array}{c}\text { Rate } \\
\left(\mathrm{g} \cdot \mathrm{ai}^{\mathrm{h}} \mathrm{ha}^{-1}\right)\end{array}$ & $\begin{array}{c}\text { Moisture } \\
(\%)\end{array}$ & $\begin{array}{c}\text { Yield } \\
\left(\mathrm{T} \cdot \mathrm{ha}^{-1}\right)\end{array}$ \\
\hline Weed-free control & & $18.7 \mathrm{a}$ & $2.63 \mathrm{ab}$ \\
Weedy control & 600 & $19.4 \mathrm{ab}$ & $0.74 \mathrm{e}$ \\
Trifluralin & 1155 & $19.4 \mathrm{ab}$ & $1.48 \mathrm{de}$ \\
Trifluralin & 810 & $19.3 \mathrm{ab}$ & $1.56 \mathrm{de}$ \\
Ethalfluralin & 1080 & $18.9 \mathrm{a}$ & $1.84 \mathrm{bcd}$ \\
Ethalfluralin & 35 & $19.1 \mathrm{ab}$ & $1.74 \mathrm{~cd}$ \\
Halosulfuron & $600+35$ & $18.9 \mathrm{a}$ & $2.70 \mathrm{a}$ \\
Trifluralin + halosulfuron & $1155+35$ & $18.8 \mathrm{a}$ & $2.49 \mathrm{abc}$ \\
Trifluralin + halosulfuron & $810+35$ & $18.7 \mathrm{a}$ & $2.55 \mathrm{abc}$ \\
Ethalfluralin + halosulfuron & $1080+35$ & $18.7 \mathrm{a}$ & $2.60 \mathrm{ab}$ \\
\hline Ethalfluralin + halosulfuron & &
\end{tabular}

${ }^{a}$ Abbreviations: PPI, preplant incorporated; WAE, weeks after white bean emergence. ${ }^{b}$ Means followed by a different letter within a column are significantly different according to a Tukey-Kramer multiple range test at $\mathrm{P}<0.05$.

Table 2. Percent visible control 4, 8 and 12 WAE of velvetleaf treated with trifluralin or ethalfluralin and halosulfuron applied PPI at Ridgetown, ON, Canada (2016-2018), a,

\begin{tabular}{ccccc}
\hline & Rate & \multicolumn{3}{c}{ Velvetleaf control (\%) } \\
\cline { 3 - 5 }$\left(\mathrm{g} \cdot \mathrm{ai} \cdot \mathrm{ha}^{-1}\right)$ & $4 \mathrm{WAE}$ & $8 \mathrm{WAE}$ & $12 \mathrm{WAE}$ \\
\hline Treatment & & 100 & 100 & 100 \\
Weed-free control & & $0 \mathrm{c}$ & $0 \mathrm{~d}$ & $0 \mathrm{c}$ \\
Trifluralin & 600 & $15 \mathrm{~b}$ & $2 \mathrm{~cd}$ & $2 \mathrm{bc}$ \\
Trifluralin & 1155 & $32 \mathrm{~b}$ & $27 \mathrm{~b}$ & $25 \mathrm{~b}$ \\
Ethalfluralin & 810 & $13 \mathrm{~b}$ & $6 \mathrm{bcd}$ & $5 \mathrm{bc}$ \\
Ethalfluralin & 1080 & $18 \mathrm{~b}$ & $14 \mathrm{bc}$ & $11 \mathrm{bc}$ \\
Halosulfuron & 35 & $76 \mathrm{a}$ & $74 \mathrm{a}$ & $69 \mathrm{a}$ \\
Trifluralin + halosulfuron & $600+35$ & $83 \mathrm{a}$ & $76 \mathrm{a}$ & $69 \mathrm{a}$ \\
Trifluralin + halosulfuron & $1155+35$ & $88 \mathrm{a}$ & $84 \mathrm{a}$ & $76 \mathrm{a}$ \\
Ethalfluralin + halosulfuron & $810+35$ & $88 \mathrm{a}$ & $76 \mathrm{a}$ & $67 \mathrm{a}$ \\
Ethalfluralin + halosulfuron & $1080+35$ & $88 \mathrm{a}$ & $79 \mathrm{a}$ & $71 \mathrm{a}$ \\
\hline
\end{tabular}

${ }^{a}$ Abbreviations: PPI, preplant incorporated; WAE, weeks after white bean emergence. ${ }^{b}$ Means followed by a different letter within a column are significantly different according to a Tukey-Kramer multiple range test at $\mathrm{P}<0.05$ 
Table 3. Percent visible control 4, 8 and 12 WAE of redroot pigweed treated with trifluralin or ethalfluralin and halosulfuron applied PPI at Exeter (2017) and Ridgetown, ON, Canada (2017). , $^{\mathrm{a}}$

\begin{tabular}{ccccc}
\hline & Rate & \multicolumn{3}{c}{ Redroot pigweed control (\%) } \\
\cline { 3 - 5 } Treatment & $\left(\mathrm{g} \cdot \mathrm{ai} \cdot \mathrm{ha}^{-1}\right)$ & $4 \mathrm{WAE}$ & $8 \mathrm{WAE}$ & $12 \mathrm{WAE}$ \\
\hline Weed-free control & & 100 & 100 & 100 \\
Weedy control & & $0 \mathrm{~b}$ & $0 \mathrm{~b}$ & $0 \mathrm{~b}$ \\
Trifluralin & 1155 & $98 \mathrm{a}$ & $99 \mathrm{a}$ & $99 \mathrm{a}$ \\
Trifluralin & 810 & $99 \mathrm{a}$ & $99 \mathrm{a}$ & $99 \mathrm{a}$ \\
Ethalfluralin & 1080 & $99 \mathrm{a}$ & $99 \mathrm{a}$ & $99 \mathrm{a}$ \\
Ethalfluralin & 35 & $98 \mathrm{a}$ & $97 \mathrm{a}$ & $97 \mathrm{a}$ \\
Halosulfuron & $600+35$ & $99 \mathrm{a}$ & $99 \mathrm{a}$ & $99 \mathrm{a}$ \\
Trifluralin + halosulfuron & $1155+35$ & $100 \mathrm{a}$ & $100 \mathrm{a}$ & $99 \mathrm{a}$ \\
Trifluralin + halosulfuron & $810+35$ & $99 \mathrm{a}$ & $99 \mathrm{a}$ & $99 \mathrm{a}$ \\
Ethalfluralin + halosulfuron & $1080+35$ & $100 \mathrm{a}$ & $100 \mathrm{a}$ & $99 \mathrm{a}$ \\
\hline Ethalfluralin + halosulfuron & & 98 &
\end{tabular}

${ }^{\mathrm{a}}$ Abbreviations: PPI, preplant incorporated; WAE, weeks after white bean emergence. ${ }^{\mathrm{b}}$ Means followed by a different letter within a column are significantly different according to a Tukey-Kramer multiple range test at $\mathrm{P}<0.05$

Table 4. Percent visible control 4, 8 and $12 \mathrm{WAE}$ of common ragweed treated with trifluralin or ethalfluralin and halosulfuron applied PPI at Exeter (2017-2018) and Ridgetown, ON, Canada (2016-2018), ${ }^{\mathrm{a}, \mathrm{b}}$

\begin{tabular}{ccccc}
\hline \multirow{2}{*}{ Treatment } & $\begin{array}{c}\text { Rate } \\
\left(\mathrm{g} \cdot \mathrm{ai} \cdot \mathrm{ha} \mathrm{a}^{-1}\right)\end{array}$ & $4 \mathrm{WAE}$ & $8 \mathrm{WAE}$ & $12 \mathrm{WAE}$ \\
\cline { 3 - 5 } & & 100 & 100 & 100 \\
Weed-free control & & $0 \mathrm{c}$ & $0 \mathrm{c}$ & $0 \mathrm{c}$ \\
Weedy control & 600 & $0 \mathrm{c}$ & $2 \mathrm{~b}$ & $13 \mathrm{~b}$ \\
Trifluralin & 1155 & $7 \mathrm{~b}$ & $7 \mathrm{~b}$ & $10 \mathrm{~b}$ \\
Trifluralin & 810 & $14 \mathrm{~b}$ & $6 \mathrm{~b}$ & $12 \mathrm{~b}$ \\
Ethalfluralin & 1080 & $20 \mathrm{~b}$ & $13 \mathrm{~b}$ & $13 \mathrm{~b}$ \\
Ethalfluralin & 35 & $96 \mathrm{a}$ & $95 \mathrm{a}$ & $95 \mathrm{a}$ \\
Halosulfuron & $600+35$ & $97 \mathrm{a}$ & $95 \mathrm{a}$ & $94 \mathrm{a}$ \\
Trifluralin + halosulfuron & $1155+35$ & $98 \mathrm{a}$ & $97 \mathrm{a}$ & $97 \mathrm{a}$ \\
Trifluralin + halosulfuron & $810+35$ & $98 \mathrm{a}$ & $97 \mathrm{a}$ & $97 \mathrm{a}$ \\
Ethalfluralin + halosulfuron & $1080+35$ & $99 \mathrm{a}$ & $97 \mathrm{a}$ & $97 \mathrm{a}$ \\
\hline Ethalfluralin + halosulfuron & & &
\end{tabular}

${ }^{\mathrm{a}} \mathrm{Abbreviations:} \mathrm{PPI,} \mathrm{preplant} \mathrm{incorporated;} \mathrm{WAE,} \mathrm{weeks} \mathrm{after} \mathrm{white} \mathrm{bean} \mathrm{emergence.}{ }^{\mathrm{b}}$ Means followed by a different letter within a column are significantly different according to a Tukey-Kramer multiple range test at $\mathrm{P}<0.05$. 
Table 5. Percent visible control 4, 8 and 12 WAE of common lambsquarters treated with trifluralin or ethalfluralin and halosulfuron applied PPI at Exeter (2017-2018) and Ridgetown, ON, Canada (2016-2018). ${ }^{\mathrm{a}, \mathrm{b}}$

\begin{tabular}{|c|c|c|c|c|}
\hline \multirow{2}{*}{ Treatment } & \multirow{2}{*}{$\begin{array}{c}\text { Rate } \\
\left(\mathrm{g} \cdot \mathrm{ai}^{-} \cdot \mathrm{ha}^{-1}\right)\end{array}$} & \multicolumn{3}{|c|}{ Common lamb's-quarters control (\%) } \\
\hline & & $4 \mathrm{WAE}$ & $8 \mathrm{WAE}$ & $12 \mathrm{WAE}$ \\
\hline Weed-free control & & 100 & 100 & 100 \\
\hline Weedy control & & $0 \mathrm{~d}$ & $0 \mathrm{~d}$ & $0 \mathrm{~d}$ \\
\hline Trifluralin & 600 & $94 \mathrm{c}$ & $89 c$ & $88 \mathrm{c}$ \\
\hline Trifluralin & 1155 & $99 \mathrm{ab}$ & $98 \mathrm{ab}$ & $98 \mathrm{ab}$ \\
\hline Ethalfluralin & 810 & $98 \mathrm{abc}$ & $98 \mathrm{ab}$ & $97 \mathrm{ab}$ \\
\hline Ethalfluralin & 1080 & $100 \mathrm{a}$ & $100 \mathrm{a}$ & $100 \mathrm{a}$ \\
\hline Halosulfuron & 35 & $96 \mathrm{bc}$ & $93 \mathrm{bc}$ & $90 \mathrm{bc}$ \\
\hline Trifluralin + halosulfuron & $600+35$ & $98 \mathrm{abc}$ & $98 \mathrm{ab}$ & $97 \mathrm{ab}$ \\
\hline Trifluralin + halosulfuron & $1155+35$ & $100 \mathrm{a}$ & $100 \mathrm{a}$ & $99 \mathrm{a}$ \\
\hline Ethalfluralin + halosulfuron & $810+35$ & $100 \mathrm{a}$ & $100 \mathrm{a}$ & $99 \mathrm{a}$ \\
\hline Ethalfluralin + halosulfuron & $1080+35$ & $100 \mathrm{a}$ & $100 \mathrm{a}$ & $100 \mathrm{a}$ \\
\hline
\end{tabular}

${ }^{a}$ Abbreviations: PPI, preplant incorporated; WAE, weeks after white bean emergence. ${ }^{\mathrm{b}}$ Means followed by a different letter within a column are significantly different according to a Tukey-Kramer multiple range test at $\mathrm{P}<0.05$.

Table 6. Percent visible control 4, 8 and 12 WAE of wild mustard treated with trifluralin or ethalfluralin and halosulfuron applied PPI at Exeter, ON, Canada (2017-2018), a,b

\begin{tabular}{|c|c|c|c|c|}
\hline \multirow{2}{*}{ Treatment } & \multirow{2}{*}{$\begin{array}{c}\text { Rate } \\
\left(\mathrm{g} \cdot \mathrm{ai}^{-} \cdot \mathrm{ha}^{-1}\right)\end{array}$} & \multicolumn{3}{|c|}{ Wild mustard control (\%) } \\
\hline & & $4 \mathrm{WAE}$ & $8 \mathrm{WAE}$ & $12 \mathrm{WAE}$ \\
\hline Weed-free control & & 100 & 100 & 100 \\
\hline Weedy control & & $0 \mathrm{~d}$ & $0 \mathrm{~d}$ & $0 \mathrm{e}$ \\
\hline Trifluralin & 600 & $2 c$ & $10 \mathrm{c}$ & $13 \mathrm{~cd}$ \\
\hline Trifluralin & 1155 & $27 \mathrm{~b}$ & $14 \mathrm{c}$ & $6 \mathrm{~d}$ \\
\hline Ethalfluralin & 810 & $27 \mathrm{~b}$ & $25 \mathrm{bc}$ & $21 \mathrm{c}$ \\
\hline Ethalfluralin & 1080 & $47 \mathrm{~b}$ & $40 \mathrm{~b}$ & $41 \mathrm{~b}$ \\
\hline Halosulfuron & 35 & 96 a & $98 \mathrm{a}$ & $100 \mathrm{a}$ \\
\hline Trifluralin + halosulfuron & $600+35$ & 99 a & 99 a & 99 a \\
\hline Trifluralin + halosulfuron & $1155+35$ & $99 \mathrm{a}$ & $100 \mathrm{a}$ & $100 \mathrm{a}$ \\
\hline Ethalfluralin + halosulfuron & $810+35$ & $100 \mathrm{a}$ & $100 \mathrm{a}$ & $100 \mathrm{a}$ \\
\hline Ethalfluralin + halosulfuron & $1080+35$ & $97 \mathrm{a}$ & $100 \mathrm{a}$ & $100 \mathrm{a}$ \\
\hline
\end{tabular}

${ }^{\mathrm{a}} \mathrm{Abbreviations:} \mathrm{PPI,} \mathrm{preplant} \mathrm{incorporated;} \mathrm{WAE,} \mathrm{weeks} \mathrm{after} \mathrm{white} \mathrm{bean} \mathrm{emergence.}{ }^{\mathrm{b}}$ Means followed by a different letter within a column are significantly different according to a Tukey-Kramer multiple range test at $\mathrm{P}<0.05$. 
Table 7. Percent visible control 4, 8 and 12 WAE of barnyardgrass treated with trifluralin or ethalfluralin and halosulfuron applied PPI at Exeter (2017) and Ridgetown, ON, Canada (2016 and 2018).,

\begin{tabular}{ccccc}
\hline & Rate & \multicolumn{3}{c}{ Barnyardgrass control (\%) } \\
\cline { 3 - 5 } Treatment & $\left(\mathrm{g} \cdot \mathrm{ai} \cdot \mathrm{ha}^{-1}\right)$ & 4 WAE & 8 WAE & $12 \mathrm{WAE}$ \\
\hline Weed-free control & & 100 & 100 & 100 \\
Weedy control & & $0 \mathrm{c}$ & $0 \mathrm{c}$ & $0 \mathrm{~b}$ \\
Trifluralin & 1155 & $990 \mathrm{a}$ & $99 \mathrm{a}$ & $99 \mathrm{a}$ \\
Trifluralin & 810 & $99 \mathrm{a}$ & $99 \mathrm{a}$ & $99 \mathrm{a}$ \\
Ethalfluralin & 1080 & $100 \mathrm{a}$ & $99 \mathrm{a}$ & $100 \mathrm{a}$ \\
Ethalfluralin & 35 & $19 \mathrm{~b}$ & $11 \mathrm{~b}$ & $0 \mathrm{~b}$ \\
Halosulfuron & $600+35$ & $98 \mathrm{a}$ & $97 \mathrm{a}$ & $97 \mathrm{a}$ \\
Trifluralin + halosulfuron & $1155+35$ & $98 \mathrm{a}$ & $99 \mathrm{a}$ & $99 \mathrm{a}$ \\
Trifluralin + halosulfuron & $810+35$ & $98 \mathrm{a}$ & $97 \mathrm{a}$ & $98 \mathrm{a}$ \\
Ethalfluralin + halosulfuron & $1080+35$ & $100 \mathrm{a}$ & $99 \mathrm{a}$ & $99 \mathrm{a}$ \\
\hline Ethalfluralin + halosulfuron & & 98 &
\end{tabular}

${ }^{a}$ Abbreviations: PPI, preplant incorporated; WAE, weeks after white bean emergence. ${ }^{b}$ Means followed by a different letter within a column are significantly different according to a Tukey-Kramer multiple range test at $\mathrm{P}<0.05$

Table 8. Percent visible control 4, 8 and 12 WAE of green foxtail treated with trifluralin or ethalfluralin and halosulfuron applied PPI at Exeter (2017-2018) and Ridgetown, ON, Canada (2016-2018), ${ }^{\mathrm{a}, \mathrm{b}}$

\begin{tabular}{ccccc}
\hline \multirow{2}{*}{ Treatment } & $\begin{array}{c}\text { Rate } \\
\left(\mathrm{g} \cdot \mathrm{ai} \cdot \mathrm{ha}^{-1}\right)\end{array}$ & $4 \mathrm{WAE}$ & $8 \mathrm{WAE}$ & $12 \mathrm{WAE}$ \\
\cline { 3 - 5 } & & 100 & 100 & 100 \\
Weed-free control & & $0 \mathrm{~d}$ & $0 \mathrm{~d}$ & $0 \mathrm{e}$ \\
Weedy control & 600 & $96 \mathrm{~b}$ & $96 \mathrm{ab}$ & $96 \mathrm{bc}$ \\
Trifluralin & 1155 & $99 \mathrm{ab}$ & $99 \mathrm{a}$ & $99 \mathrm{ab}$ \\
Trifluralin & 810 & $99 \mathrm{ab}$ & $99 \mathrm{a}$ & $99 \mathrm{ab}$ \\
Ethalfluralin & 1080 & $99 \mathrm{ab}$ & $99 \mathrm{a}$ & $100 \mathrm{a}$ \\
Ethalfluralin & 35 & $23 \mathrm{c}$ & $14 \mathrm{c}$ & $3 \mathrm{~d}$ \\
Halosulfuron & $600+35$ & $96 \mathrm{~b}$ & $95 \mathrm{~b}$ & $94 \mathrm{c}$ \\
Trifluralin + halosulfuron & $1155+35$ & $98 \mathrm{ab}$ & $98 \mathrm{ab}$ & $98 \mathrm{abc}$ \\
Trifluralin + halosulfuron & $810+35$ & $98 \mathrm{ab}$ & $98 \mathrm{ab}$ & $98 \mathrm{abc}$ \\
Ethalfluralin + halosulfuron & $1080+35$ & $100 \mathrm{a}$ & $99 \mathrm{a}$ & $99 \mathrm{ab}$ \\
\hline Ethalfluralin + halosulfuron & &
\end{tabular}

${ }^{\mathrm{a}} \mathrm{Abbreviations:} \mathrm{PPI,} \mathrm{preplant} \mathrm{incorporated;} \mathrm{WAE,} \mathrm{weeks} \mathrm{after} \mathrm{white} \mathrm{bean} \mathrm{emergence.}{ }^{\mathrm{b}}$ Means followed by a different letter within a column are significantly different according to a Tukey-Kramer multiple range test at $\mathrm{P}<0.05$. 
long equipped with 4 ultra-low drift (ULD 120-02, Pentair-Hypro, New Brighton, Minnesota) nozzles spaced $0.5 \mathrm{~m}$ apart producing a spray width of $2.0 \mathrm{~m}$.

White bean visible injury was assessed 2, 4 and 8 weeks after emergence (WAE) and weed control efficacy was assessed visually on a scale of 0 (no injury/control) to $100 \%$ (total plant necrosis/weed control) 4,8 and 12 WAE. White bean yield was determined by harvesting the middle two rows of each plot with a plot combine at maturity. White bean yield was adjusted to $18 \%$ seed moisture content.

\subsection{Statistical Analyses}

The GLIMMIX procedure in SAS [13] was utilized for data analysis, with herbicide treatment as the fixed effect and year-location combinations, replicate within environment and environment by treatment interaction as the random effects. Potential distributions were assessed using residual plots, fit statistics and the Shapiro-Wilk statistic to find the most appropriate distribution or transformation. Analysis was performed on the model scale, with least square means presented on the data scale. Treatment differences were determined at a significance level of 0.05 , after pairwise comparisons were subjected to Tukey's adjustment. The Gaussian distribution and identity link were used for percent control of velvetleaf ( 4,8 and $12 \mathrm{WAE}$ ), wild mustard (12 WAE), white bean moisture at harvest and yield. All other percent weed control data were arcsine square-root transformed prior to analysis, and back-transformed for presentation. Treatments with zero variance were excluded from the analysis and included the weedy control (assigned a value of zero) and the weed-free control (assigned a value of 100) for percent weed control. Comparisons of each treatment with the value zero were still conducted and differences identified using the $P$ values from the LSMEANS output.

\section{Results and Discussion}

\subsection{Crop Injury}

At 2, 4 and $8 \mathrm{WAE}$, there was no white bean injury from the herbicide treatments evaluated (data not presented). There was a delay in white bean maturity due to weed interference as indicated by increased seed moisture content at harvest (Table 1). Weeds interference decreased white bean seed yield $72 \%$ (Table 1). Generally, white bean yield reflected the level of weed control provided by the herbicide treatments evaluated. There was no difference in white bean seed yield between trifluralin and ethalfluralin. Weed presence decreased seed yield $44 \%$ - 45\% with trifluralin and 30\% - 41\% with ethalfluralin (Table 1). Weed interference with halosulfuron applied alone decreased seed yield $34 \%$ (Table 1). In contrast, decreased weed interference with trifluralin and ethalfluralin applied in combination with halosulfuron resulted in white bean seed yield that was similar to the weed-free control. 
These results are similar to other studies that have shown little white bean injury with trifluralin and trifluralin + halosulfuron in white bean. Soltani et al. 2014 [4] found no injury with trifluralin applied PPI alone and 1\% injury with trifluralin + halosulfuron applied PPI in white bean [4]. In other studies, halosulfuron, applied PPI was shown to be safe for use on most market classes of dry beans except for azuki, mung, and snap bean [14] [15] [16] [17]. In another study, halosulfuron caused $8 \%$ injury and reduced seed yield $7 \%$ in snap bean [14].

\subsection{Weed Control}

Trifluralin (600 g.ai.ha ${ }^{-1}$ ), applied PPI, provided 2\% - 15\%, 96\% - 98\%, 0\% $13 \%, 88 \%-94 \%, 2 \%-13 \%, 97 \%-98 \%$ and $96 \%$ control of velvetleaf, redroot pigweed, common ragweed, common lambsquarters, wild mustard, barnyardgrass and green foxtail, respectively (Tables 2-8). Ethalfluralin (810 g.ai $\cdot \mathrm{ha}^{-1}$ ) provided similar control of velvetleaf, redroot pigweed, barnyardgrass and green foxtail and better control of common lambsquarters at 8 and $12 \mathrm{WAE}$, common ragweed at $4 \mathrm{WAE}$, and wild mustard at 4 WAE (Tables 2-8).

At 4, 8 and 12 WAE, trifluralin (1155 g.ai $\cdot \mathrm{ha}^{-1}$ ), applied PPI, provided 25\% $32 \%, 98 \%-99 \%, 7 \%-10 \%, 98 \%-99 \%, 6 \%-27 \%, 99 \%$ and $99 \%$ control of velvetleaf, redroot pigweed, common ragweed, common lambsquarters, wild mustard, barnyardgrass and green foxtail, respectively. Ethalfluralin (1080 g.ai $\cdot \mathrm{ha}^{-1}$ ) provided similar control of velvetleaf, redroot pigweed, common ragweed, common lambsquarters, barnyardgrass and green foxtail and better control of wild mustard at 8 and 12 WAE (Tables 2-8).

At 4, 8 and $12 \mathrm{WAE}$, halosulfuron (35 g.ai $\cdot \mathrm{ha}^{-1}$ ), applied PPI, provided $69 \%$ $76 \%, 97 \%-98 \%, 95 \%-96 \%, 90 \%-96 \%, 96 \%-100 \%, 0 \%-19 \%$ and $3 \%-23 \%$ control of velvetleaf, redroot pigweed, common ragweed, common lambsquarters, wild mustard, barnyardgrass and green foxtail, respectively (Tables 2-8).

At 4, 8 and $12 \mathrm{WAE}$, trifluralin $\left(600 \mathrm{~g} \cdot \mathrm{ai} \cdot \mathrm{ha}^{-1}\right)+$ halosulfuron $\left(35 \mathrm{~g} \cdot \mathrm{ai} \cdot \mathrm{ha} \mathrm{a}^{-1}\right)$, applied PPI, provided 69\% - 83\%, 99\%, 94\% - 97\%, 97\% - 98\%, 99\%, 97\% - 98\% and $94 \%-96 \%$ control of velvetleaf, redroot pigweed, common ragweed, common lambsquarters, wild mustard, barnyardgrass and green foxtail, respectively (Tables 1-7). Ethalfluralin (810 g.ai $\left.\cdot \mathrm{ha}^{-1}\right)+$ halosulfuron (35 g.ai $\cdot \mathrm{ha}^{-1}$ ) provided similar control of the same weed species (Tables 2-8).

At 4, 8 and 12 WAE, trifluralin (1155 g.ai $\left.\cdot \mathrm{ha}^{-1}\right)+$ halosulfuron (35 g.ai $\cdot \mathrm{ha}^{-1}$ ), applied PPI, provided 76\% - 88\%, 99\% - 100\%, 97\% - 98\%, 99\% - 100\%, 99\% $100 \%, 98 \%-99 \%$ and $98 \%$ control of velvetleaf, redroot pigweed, common ragweed, common lambsquarters, wild mustard, barnyardgrass and green foxtail, respectively. Ethalfluralin (1080 g.ai $\left.\cdot \mathrm{ha}^{-1}\right)+$ halosulfuron $\left(35 \mathrm{~g} \cdot \mathrm{ai} \cdot \mathrm{ha}^{-1}\right)$ provided similar control of the same weed species (Tables 2-8).

The results from this study are similar to other research in which trifluralin applied PPI controlled redroot pigweed $72 \%$ - 98\%, common ragweed $9 \%-28 \%$, common lambsquarters $60 \%-92 \%$, wild mustard $11 \%-44 \%$, and green foxtail 
$94 \%-100 \%$ in dry bean [4] [5] [15] [18]. In other studies, halosulfuron applied PPI controlled redroot pigweed 83\% - 100\%, common ragweed 95\% - 99\%, common lambsquarters $96 \%$ - 100\%, wild mustard $99 \%-100 \%$, and green foxtail 47\% - 59\% [4] [5] [18].

\section{Conclusions}

Trifluralin and ethalfluralin applied PPI alone or in combination with halosulfuron caused no visible injury in white bean at rates evaluated. There was delayed white bean maturity due to weed interference as indicated by increased seed moisture content at harvest. Weeds presence decreased white bean seed yield $72 \%$. Generally, white bean yield reflected the level of weed control provided by the herbicide treatments evaluated. There was no difference in white bean seed yield between trifluralin and ethalfluralin. Decreased weed interference with trifluralin and ethalfluralin applied in combination with halosulfuron resulted in white bean seed yield that was similar to the weed-free control. Trifluralin and ethalfluralin applied PPI alone did not provide adequate control of velvetleaf, common ragweed and wild mustard, but provided good to excellent control of redroot pigweed, common lambsquarters, barnyardgrass and green foxtail. Trifluralin and ethalfluralin provide similar control of velvetleaf, redroot pigweed, barnyardgrass and green foxtail control, however, ethalfluralin is slightly more efficacious on common ragweed, common lambsquarters and wild mustard. Halosulfuron applied PPI alone provided inadequate control of barnyardgrass and green foxtail, fair control of velvetleaf and good to excellent control of redroot pigweed, common ragweed, common lambsquarters and wild mustard. Trifluralin and ethalfluralin applied PPI in combination with halosulfuron provided good to excellent control of velvetleaf, redroot pigweed, common ragweed, common lambsquarters, wild mustard, barnyardgrass and green foxtail. This study concludes that trifluralin or ethalfluralin co-applied with halosulfuron can be safely used for broad-spectrum control of annual grass and broadleaf weeds in white bean production in Ontario.

\section{Acknowledgements}

This study was funded in part by Ontario Bean Growers (OBG).

\section{Conflicts of Interest}

The authors declare that there is no conflict of interest regarding the publication of this paper.

\section{References}

[1] Soltani, N., Dille, J.A., Burke, I.C., Everman, W.J., VanGessel, M.J., Davis, V.M. and Sikkema, P.H. (2016) Potential Corn Yield Losses from Weeds in North America. Weed Technology, 30, 979-984. https://doi.org/10.1614/WT-D-16-00046.1

[2] Ontario Ministry of Agriculture and Food and Rural Affairs (OMAFRA) (2010) 
Area, Yield, Production and Farm Value of Specified Field Crops, Ontario, 2012-2017.

http://www.omafra.gov.on.ca/english/stats/crops/estimate_new.htm\#metric

[3] Malik, V.S., Swanton, C.J. and Michaels, T.E. (1993) Interaction of White Bean (Phaseolus vulgaris) Cultivars, Row Spacing, and Seeding Density with Annual Weeds. Weed Science, 41, 62-68. https://doi.org/10.1017/S0043174500057593

[4] Soltani, N., Nurse, R.E., Shropshire, C. and Sikkema, P.H. (2014) Weed Control in White Bean with Various Halosulfuron Tankmixes. Advances in Agriculture, 2014, Article ID: 391634. https://doi.org/10.1155/2014/391634

[5] Soltani, N., Nurse, R.E., Shropshire, C. and Sikkema, P.H. (2014) Weed Control with Halosulfuron Applied Preplant Incorporated, Preemergence or Postemergence in White Bean. Agricultural Science, 5, 875-881.

https://doi.org/10.4236/as.2014.510094

[6] Wilson, R.G. (1993) Wild Proso Millet (Panicum miliaceum) Interference in Dry Beans (Phaseolus vulgaris). Weed Science, 41, 607-610. https://doi.org/10.1017/S0043174500076396

[7] OMAFRA, Ontario Ministry of Agriculture and Food and Rural Affairs (2020) Guide to Weed Control, Publication 75. Toronto, 1-396.

[8] Shaner, D. (2014) Herbicide Handbook, Tenth Edition. Weed Science Society of America, Champaign, $458 \mathrm{p}$.

[9] NCBI, National Center for Biotechnology Information (2020) PubChem Compound Summary for CID 5569, Trifluralin.

https://pubchem.ncbi.nlm.nih.gov/compound/Trifluralin

[10] Gowan (2020) EDGE Granular Herbicide. https://ca.gowanco.com/products/edge-granular

[11] Beckie, H.J. and Morrison, I.N. (1993) Effect of Ethalfluralin and Other Herbicides on Trifluralin-Resistant Green Foxtail (Setaria viridis). Weed Technology, 7, 6-14. https://doi.org/10.1017/S0890037X00036782

[12] Zimdahl, R.L., Cattizone, P. and Butcher, A.C. (1984) Degradation of Pendimethalin in Soil. Weed Science, 32, 408-412. https://doi.org/10.1017/S004317450005921X

[13] SAS Institute Inc. (2016) Base SAS ${ }^{\circledast} 9.4$ Procedures Guide: Statistical Procedures, Fifth Edition. SAS Institute Inc., Cary.

[14] Silvey, B.D., Mitchem, W.E., Macrae, A.W. and Monks, D.W. (2006) Snap Bean (Phaseolus vulgaris) Tolerance to Halosulfuron PRE, POST, or PRE Followed by POST. Weed Technology, 20, 873-876. https://doi.org/10.1614/WT-05-046.1

[15] Soltani, N., Nurse, R.E., Van Eerd, L.L., Shropshire, C. and Sikkema, P.H. (2010) Weed Control, Environmental Impact and Profitability with Trifluralin plus Reduced Doses of Imazethapyr in Dry Bean. Crop Protection, 29, 364-368. https://doi.org/10.1016/j.cropro.2009.07.011

[16] Soltani, N., Nurse, R. and Sikkema, P.H. (2013) Weed Management in White Bean with Postemergence Herbicide Tankmixes. Canadian Journal of Plant Science, 93, 669-674. https://doi.org/10.4141/cjps2012-273

[17] Stewart, C.L., Nurse, R.E., Gillard, C.L. and Sikkema, P.H. (2010) Tolerance of Adzuki Bean to Preplant-Incorporated, Pre-Emergence, and Post-Emergence Herbicides in Ontario, Canada. Weed Biology and Management, 10, 40-47. https://doi.org/10.1111/j.1445-6664.2010.00365.x

[18] Li, Z., Van Acker, R., Robinson, D.E., Soltani, N. and Sikkema, P.H. (2016) Halo- 
N. Soltani et al.

sulfuron Tankmixes Applied Preplant Incorporated for Weed Control in White Bean (Phaseolus vulgaris L.). Canadian Journal of Plant Science, 96, 81-88.

https://doi.org/10.1139/cjps-2015-0124 